\title{
A Distributed Community-based Routing Algorithms for Opportunistic Networks
}

\author{
RenZhi ${ }^{1}$, Cao HongWei ${ }^{2}$, Zhang Yong ${ }^{3}$, Zhao YaNan ${ }^{4}$ \\ School of Chongqing University of Posts and Telecommunications, Chongqing 400065, China

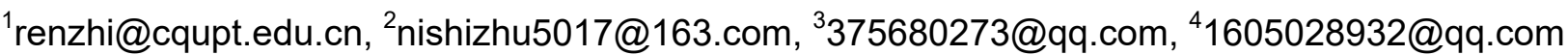

Keywords: Opportunistic networks, routing algorithms, distributed communities, message transmission, cross-layer listening mechanism

\begin{abstract}
According to the problem that there are redundant control overheads in community partitioning phase and the large transmission delay and the low success rate in transmission phase in the community-based opportunistic network, we propose EDCRA (Efficient Distributed Community-based Routing Algorithms for opportunistic networks). By distinguishing the community of the encounter node is single-node community or multi-node community, EDCRA simplify the interaction flow of the encounter node merging into other community in the community partitioning phase. And through the efficient cross-layer listening mechanism, the node carrying message find eligible forwarding nodes in its neighbor nodes in the data transmission phase, so that the message can be forwarded to the destination community more efficiently. Simulation results show that the performance of EDCRA algorithm is higher than that of CR and Bubble Rap in terms of control overhead, average end-to-end delay and transmission success rate.
\end{abstract}

\section{Introduction}

Opportunistic networks (OppNets) is a kind of mobile self-organizing networks which does not necessarily existing complete and reliable communication path between source node and destination node. The opportunity of intermittent connection between nodes will be used to realize delivering message. OppNets has great research value and application prospect[1-3]. And if the node in the OppNets move along with the movement of people, then the node has social features and the phenomenon of "small world, big world"[4] present out. According to the node movement model, the community-based opportunistic networks is created. This kind of networks make forwarding more purposeful, message replications and network resources used more less[5]. Currently, the representative routing algorithm based on distributed community in OppNets has a lot, such as CR[6] 、 SNBTR(Social Network-Based Trustworthy Routing)[7]、FBR(Friendship Based Routing)[8]、 LPBSMR (Location Preference Based Social-aware Multicast Routing)[9]、SMART (Social and Mobile Aware Routing strategy for disruption-Tolerent networks)[10], etc.

Because of high active node encountering more node, $\mathrm{CR}$ algorithm choose nodes with high global center or local center as relay nodes when message transmission in inter-community and intra-community respectively. Message delivering to destination by those relay node will reduce messages replications in the network. But two aspects about CR need to be improve: the interaction processes of a new node joining a community exist redundant messages and the message carrying node can monitoring the transmission of neighbor node in cross-layer way. Based on the comprehensive analysis of the above algorithm, EDCRA algorithm is proposed in this paper.

\section{System model and problem description}

Based on the analysis of community segmentation process and the messaging process of CR algorithm, we found some problems that:

(1) The process of a community merging a new node shown in Fig. 1. When node $i$ on behalf of its community to merge the meeting node $j$, if the community of $j$ 's only has one node and $j$ want join $C_{i}, i$ no need to send the request message, and $j$ no need to response to that message. $i$ can send 
its community diagram directly, and $j$ answer its social relation, thus reducing the number of control messages.

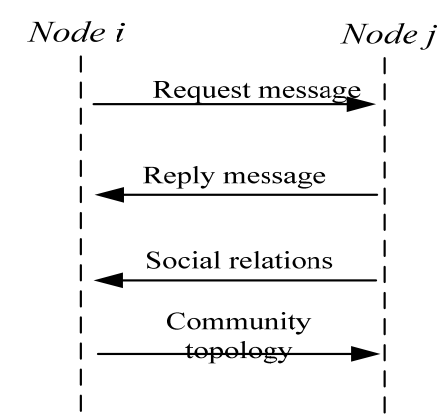

Fig. 1 The interaction flow diagram of $\mathrm{Ci}$ merging $\mathrm{j}$

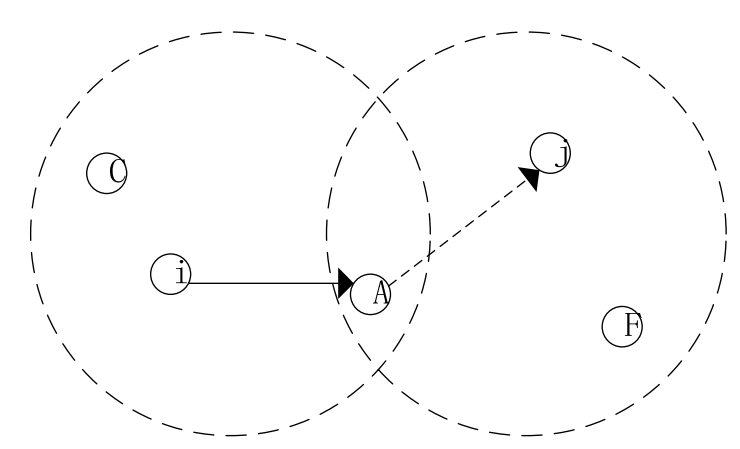

Fig. 2 Cross-layer surveillance mechanism

(2) In general, after node $i$ carrying message $m$ meet node $C$ and establish neighbor relationship, they will interchange message digest and determine whether to forward message to the other party by comparing their center degree on the message or social relation weight. As shown in Fig. 2, if the node $i$ listen to $A$ sending or receiving messages, $i$ will know the neighbor nodes of A. If $j$ is $A$ 's neighbors and the destination or in destination community of the message $m$, then $i$ still send message $m$ to $C$ and not to $A$, thus will decrease the success rate of forward and increase the messages delay and replications.

\section{EDCRA algorithm}

\subsection{Mechanism of EDCRA}

1) Low overhead community partition mechanism

Node $i$ and node $j$, for example, when $i$ represents its community $C_{i}$ to merge $j, i$ determine whether $j$ in a single node community first of all, then

a. If $\mathrm{j}$ is in single node community and the interaction process between $\mathrm{i}$ and $\mathrm{j}$ as shown in Fig. 3, $\mathrm{i}$ directly send its community topology rather than a request message to $\mathrm{j}$. After received the message, $j$ will directly response its social relationships topology to $i$. In this way, EDCRA educe the control overhead of sending request and reply messages, and to reduce the communication time of requesting and waiting for a response.

b. If $j$ isn't in a single node community, the interactive process shown in Fig. 4. $i$ send a request message to $j$, then $j$ receives the request and calculate the correlation degree $R$ (from [11])as follows:

$$
R_{(j, C)}=\sum_{\forall k \in C} W_{j}^{k}
$$

where $R_{(j, C)}$ is the correlation degree of $j$ and community $C$.

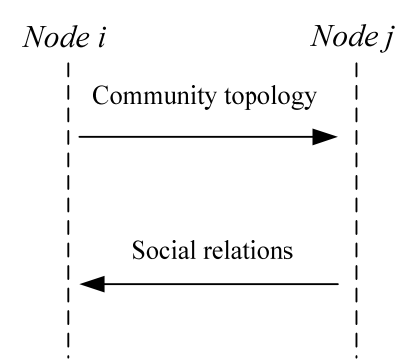

Fig. 3 Interaction process when $\mathrm{j}$ is in single node community

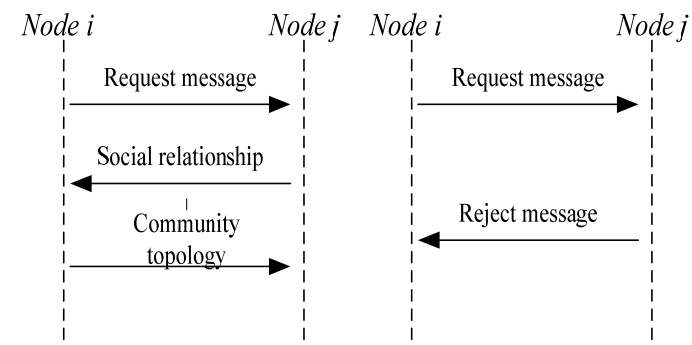

Fig. 4 Interaction process when $\mathrm{j}$ is in more node community:(a)j agree to be merged; (b) $j$ refuse to be merged.

According to the result of $R, j$ decides whether to join the community $C_{i}$ :

a. When the correlation degree of $j$ and $C_{i}$ is greater than that of $j$ and $C_{j}, j$ will send its social relations message list to $i$ and be merged. In this way, $j$ no need response a reply message to $i$, thus the control overhead and the communication time can be reduced. 
b. When the correlation degree of $j$ and $C_{i}$ is smaller, $j$ refused to be merged and will reply a refuse message to $i$.

2) Efficient cross-layer monitoring mechanism

Cross-layer monitoring mechanism is that the node carrying message monitor its neighbor sending and receiving messages to find some useful message and assist message transmission.

As shown in Fig. 2, $i$ carrying message $m$ move randomly and broadcast Hello information to find its neighbors. When the neighbor relationship is determined and $i$ monitor data or control frame send or received by its neighbor $(A)$ at the MAC layer, $i$ will extract MAC address from that frame and share information to the network layer through the across-layer way, then get the destination IP address and so as to know the neighbor of $A$. When the neighbor nodes of $A$ is the destination or in the same community with destination of $m, i$ will send $m$ to $A$ even its global center higher, and then A forward $m$ to destination.

\subsection{Algorithm operating}

1) Community partition method

Adopting a distributed community partition algorithm, EDCRA use the weights of social relations to measure the close degree between nodes, and community module degree(Q) is adopted to represent the of close degree of community structure. Community partition steps are as follows:

Step 1: In the initialization phase, opportunity network is divided into $\mathrm{n}$ parts and each node represents a community. Node number is the same as community number and $\mathrm{Q}=0$;

Step 2: After the warm-up time $\Delta t$, each node calculates its social relation weights. At the same time, them contain the neighbor node into its community in turn and calculated the corresponding Q.

Step 3: Each node will select the node making its Q increases the most and merge the node to its community. Merging process: if the node $i$ on behalf of $C_{i}$ to merge $j$, then

(1) If $C_{j}$ only has $\mathrm{j}$, then the message interaction process between $i$ and $j$ is shown in Fig. 3 .

(2) If $C_{j}$ has many nodes and $R_{(j, C j)}<R_{(j, C i)}$ then the message interaction process between $i$ and $j$ is shown in Fig.4(a). If $R_{(j, C j)}>R_{(j, C i)}$, the interaction process is shown in Fig.4(b).

Step 4: After complete the merger, $i$ and $j$ update their module value.

2) Data transmission process

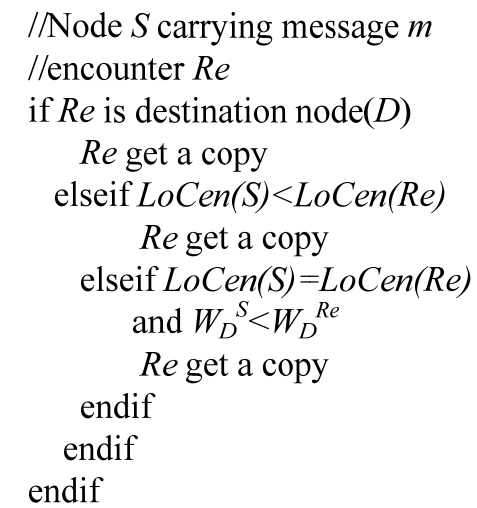

Fig. 5 Intra-community communication

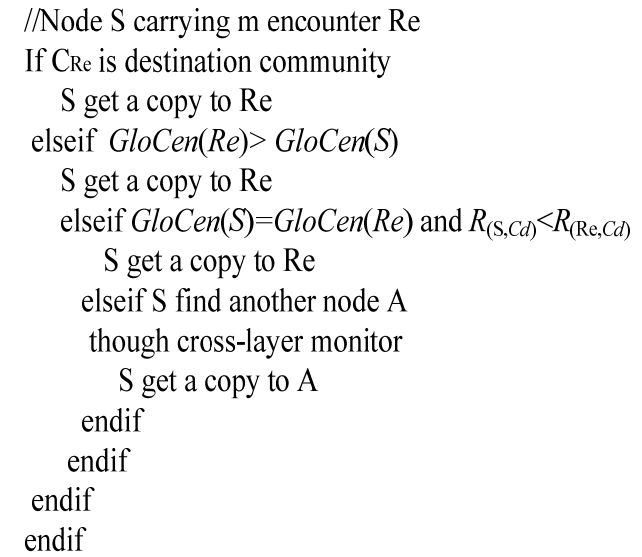

Fig. 6 Inter-community communication

\section{Simulation and results analysis}

In this paper, EDCRA, CR and Bubble Rap [12] are simulated and compared the performance indexes as follow using ONE simulator.

1) Normalized control overhead

It can be seen from Fig. 5 that EDCRA has lower normalized control overhead than the other two algorithms, because the former optimizes the interaction flow of nodes merging into the community, reduces the sending of request messages and response messages, and reduces the redundancy control overhead. 
2) Message transmission success rate

It can be seen from Fig. 6 that EDCRA is superior to the CR and Bubble Rap algorithms in the success rate of message transmission. The main reason is that EDCRA can send message to the destination node or the node which is more helpful for message transmission by listening to the neighbor node, which makes the message forwarding more effective .

3) Average end-to-end delay

It can be seen from Fig.7 that EDCRA algorithm has better end-to-end delay than other algorithms. This is because EDCRA makes full use of neighbor nodes to forward messages by listening to the messages sent and received by the nodes in the overlapped area of the communication, thus reducing the time for message transmission to the destination node.

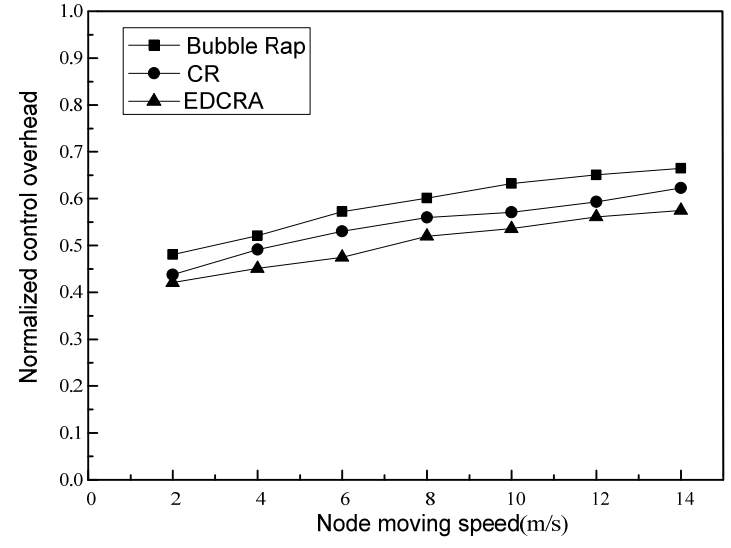

Fig. 7 Effect of node moving speed on normalized control overhead

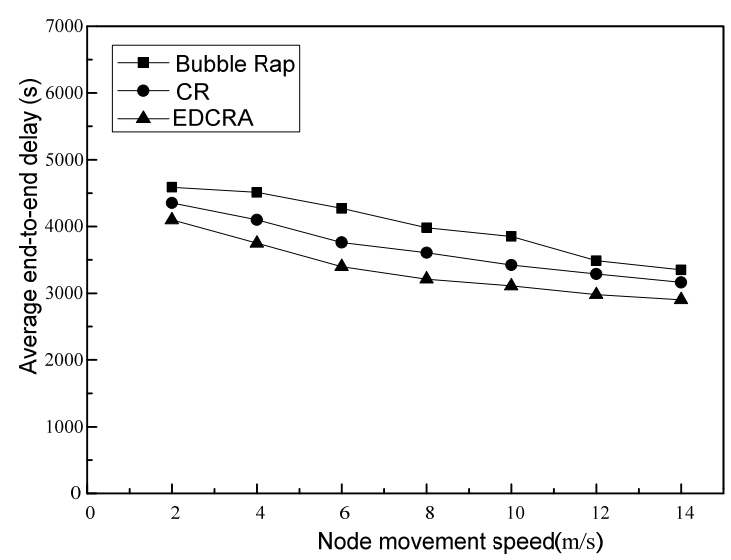

Fig. 9 Effect of node speed on average end-to-end delay

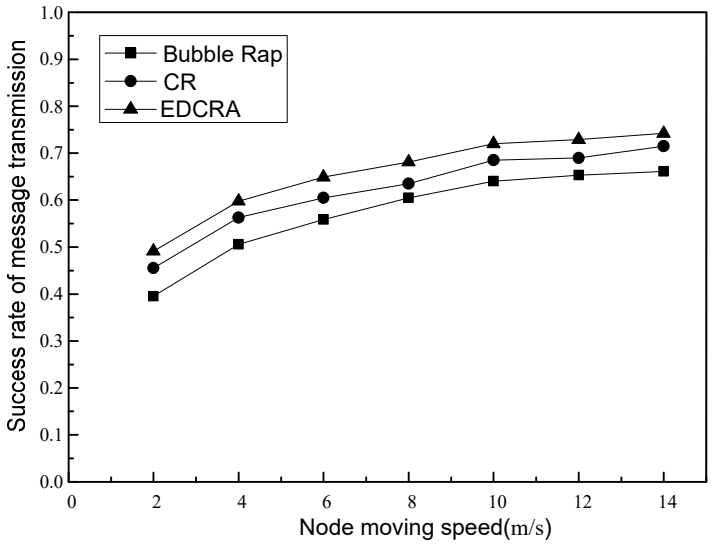

Fig. 8 Node moving speed on the success rate of the message transmission

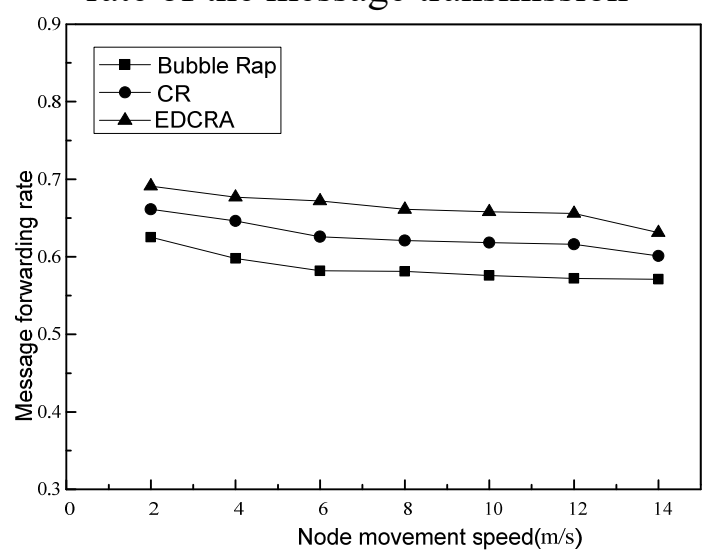

Fig. 10 Effect of node mobility speed on message forwarding efficiency

4) Message forwarding efficiency

It can be seen from Fig. 8 that the EDCRA algorithm has the highest message forwarding efficiency. The main reason is that EDCRA uses the method of cross-layer monitoring method to send the message to the destination node or the destination community with the help of the neighbor node, which reduces the unwanted propagation of the message in the network and makes the message forwarding more purposeful, thus improving the forwarding efficiency of the message.

\section{Conclusion}

In order to solve the problem that the existing community-based routing algorithm $\mathrm{CR}$ has the redundant control overhead in the community partitioning stage and not take full advantage of the node forwarding message in the overlapped area of the communication to affect the transmission success rate and increase the transmission delay, then we proposed EDCRA algorithm. Through EDCRA compared with the CR and Bubble Rap, the interactive flow of the community partitioning phase is optimized and the messages are forwarded by the neighbor nodes through an efficient cross-layer listening mechanism. These new mechanisms effectively reduce the network control 
overhead and message transmission delay, increases the transmission success rate, improve the network performance.

Fund project: National Natural Science Foundation of China (61379159); Chongqing City Foundation and Frontier Research Program Key Project (cstc2015jcyjBX0085)

\section{References}

[1] Zhi Ren, Yong Huang, Qianbin Zhen. Routing protocols for opportunistic networks[J]. Journal of Computer Applications, 2010, 30(3): 723-728.

[2] Jian Yang, Zongming Fei, Ju Shen. Hole detection and shape-free representation and double landmarks based geographic routing in wireless sensor networks[J]. Digital Communications and Networks, 2015, 9(1):433-46.

[3] Syed Rehan Afzal, Sender Stuijk, Majid Nabi, Twan Basten. Effective link quality estimation as means to improved end-to-end packet delivery in high traffic mobile ad hoc networks[J]. Digital Communications and Networks, 2016.

[4] Yunfei Bai, Yuanan Liu, etc. Routing with synthetical estimation of contact metrics and forwarding constraint in delay tolerant social networks[J]. Journal of Jilin University, 2013, 43(4): 1082-1090.

[5] Yahong Liu, Yuan Gao, etc. Community-based message transmission scheme in opportunistic social networks[J]. Journal of Computer Applications, 2013, 33(5): 1212-1216.

[6] Xuebin Ma, Xiangyu Bai. A community-based routing algorithm for opportunistic networks[C]// Proceedings of the IEEE conference on Ubiquitous and Future Networks:Da Nang: IEEE Press, 2013: 701-706.

[7] Haizheng Yu, Jianfeng Ma, Hong Bian. Social network-based trustworthy routing in delay tolerant networks[J]. Journal on Communications, 2010.12, 31( 2): 20-26.

[8] Bulut E, Szymanski B K. Friendship Based Routing in Delay Tolerant Mobile SocialNetworks[C]// Proceedings of the IEEE conference on Global Telecommunications. Mami: IEEE Press, 2010:1-5.

[9] Jiaxu Chen, Yazhe Tang, etc. Design of a Social-Aware Multicast Routing Protocol Based on Location Preference in Delay Tolerant Networks[J]. Journal of xi'an jiaotong university, 2012, 48(6):13-18.

[10]Zhu Konglin, Li Wenzhong, Fu Xiaoming. SMART: A social-and mobile-aware routing strategy for disruption-tolerant networks[J]. IEEE Transactions on Vehicular Technology, 2014, 63(7): 3423-3434.

[11]Ma Xuebin, Bai Xiangyu. A community-based routing algorithm for opportunistic networks[C]// Proceedings of the IEEE conference on Ubiquitous and Future Networks: Da Nang: IEEE Press, 2013: 701-706.

[12]Pan Hui, Crowcroft J, Eiko Y. Bubble Rap: social-based forwarding in delay-tolerant networks[J]. IEEE Transactions on Mobile Computing, 2011, 10(11): 1576-1589. 